\title{
Numerical investigation on three-dimensional shock wave reflection over two perpendicularly intersecting wedges
}

\author{
Y. Yang $\cdot$ H. Teng $\cdot$ Z. Jiang $\cdot$ K. Takayama
}

Received: 14 January 2011 / Revised: 26 May 2011 / Accepted: 7 December 2011 / Published online: 29 December 2011 (C) Springer-Verlag 2011

\begin{abstract}
The three-dimensional (3D) shock wave reflections over two perpendicularly intersecting wedges are numerically studied in this paper, using the finite volume method which is based on the MUSCL-Hancock interpolation technique and self-adaptive unstructured mesh. Two kinds of 3D Mach stem structures are demonstrated by the numerical simulations for different shock Mach numbers and wedge angles. A four-shock or three-shock wave configuration appears in the vicinity of the corner of the wedges.
\end{abstract}

Keywords Shock wave reflection - Three-dimensional Mach stem $\cdot$ Perpendicularly intersecting wedges .

Numerical simulation

\section{Introduction}

Shock wave reflection is a fundamental topic in aerodynamic research and engineering applications. The phenomena of shock wave reflection were first investigated by Mach [1] in 1870s and the well-known 'Mach reflection (MR)' was later named after him. Different types of MR configurations were further demonstrated by von Neumann [2,3] in 1940s. In recent decades, shock wave reflections have been studied systematically, e.g., shock wave reflections over wedges [4-6],

Communicated by B.W. Skews.

Y. Yang $(\varangle) \cdot H$. Teng $\cdot$ Z. Jiang

Key Laboratory of the High-Temperature Gas Dynamics, Institute of Mechanics, Chinese Academy of Sciences, Beijing 100190, China

e-mail: yy@imech.ac.cn

K. Takayama

Shock Wave Interdisciplinary Application Division, Institute of Fluid Science, Tohoku University,

Sendai 980-8577, Japan the hysteresis phenomena in steady shock wave reflections [7-10], and the application of new experimental facilities [11]. However, the previous results are mainly on two-dimensional (2D) cases, and 3D shock wave reflection has not yet been investigated widely. This is mainly because the wave structures induced by 3D shock wave reflection are usually very complicated and thus difficult to be visualized clearly by the traditional visualization techniques.

Shock wave reflection over two perpendicularly intersecting wedges is schematically shown in Fig. 1. This configuration of 3D shock reflection was first studied by Meguro et al. [12] experimentally, numerically and analytically. The 3D Mach stem was observed as well as its existence criterion according to the reflection types over each wedge, i.e., MR or regular reflection (RR). It was found that the 3D Mach stem definitely occurs for the MR-MR interaction, possibly occurs for the MR-RR interaction, but never occurs for the RR-RR interaction. The critical condition for whether or not the 3D Mach stem appears in the MR-RR interaction was analytically derived by the 2D theory of oblique shock wave reflection. In the case, as depicted in Fig. 2, the 2D Mach stem on the vertical wedge was assumed to be two-dimensionally reflected over the horizontal wedge. Here, $M_{\mathrm{m}^{\prime}}$ denotes the Mach number of the Mach stem and $\theta_{\mathrm{m}}$ is the angle between the intersecting line of the two wedges and the horizontal wall of the shock tube. $\theta_{\mathrm{m}}$ corresponds to the inclination angle for the reflection of the Mach stem $M_{\mathrm{m}^{\prime}}$ over the horizontal wedge. $M_{\mathrm{m}^{\prime}}$ and $\theta_{\mathrm{m}}$ can be calculated by geometry relations:

$\theta_{\mathrm{m}}=\arctan (\tan \alpha \cos \beta)$

$M_{\mathrm{m}^{\prime}}=M_{\mathrm{S}} \cos \chi_{\beta} / \cos \left(\chi_{\beta}+\beta\right)$

where $\chi_{\beta}$ is the triple point trajectory angle of the Mach reflection over the vertical wedge. If the assumed $2 \mathrm{D}$ 


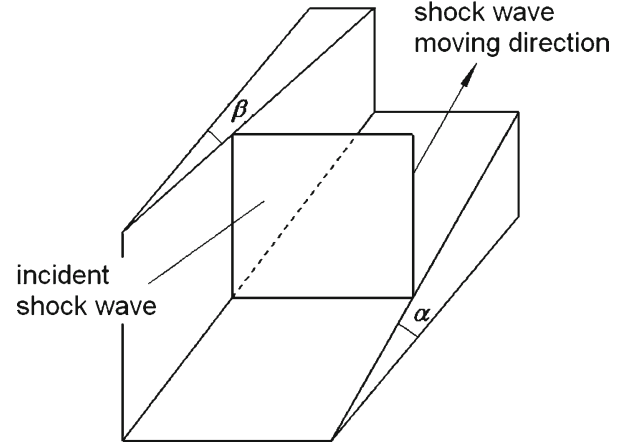

Fig. 1 Shock wave reflection over two perpendicularly intersecting wedges

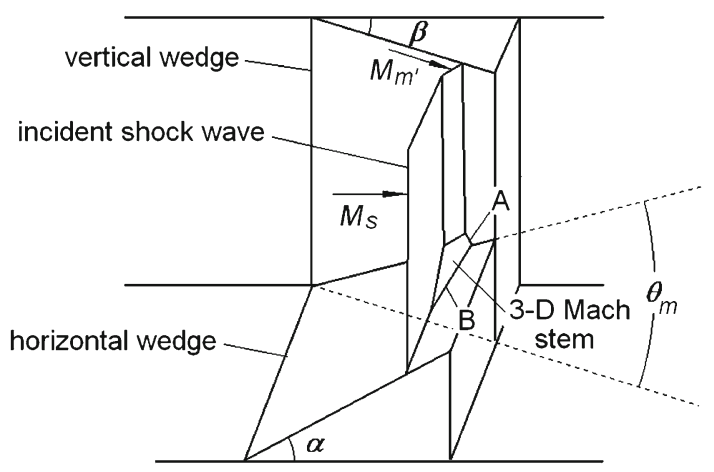

Fig. 2 Regular-Mach reflection interaction

reflection forms a Mach stem, namely A, then the Mach stems $\mathrm{A}$ and $\mathrm{B}$ would interact with each other and eventually result in a 3D Mach stem.

The 3D shock wave reflections and the detailed interaction configurations are further investigated in this paper. Two kinds of 3D Mach stems and several types of shock wave reflection configurations are figured out. The existence criterion of the 3D Mach stem deduced from the 2D theory of shock wave reflection is re-examined using the computational results.

\section{Governing equations and numerical methods}

Assuming that viscosity effects on shock wave reflection are negligible, the governing equations are the hyperbolic system of three-dimensional conservation laws in Cartesian coordinates, which can be written as:

$\frac{\partial U}{\partial t}+\frac{\partial F}{\partial x}+\frac{\partial G}{\partial y}+\frac{\partial H}{\partial z}=0$

where $U, F, G$ and $H$ denote the unknown variables and fluxes in the $x$-, $y$ - and $z$-directions, respectively:

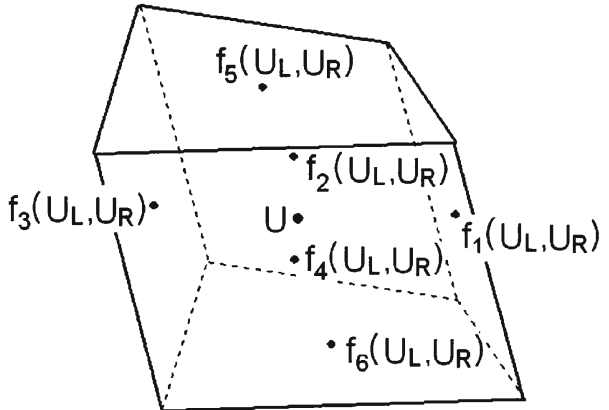

Fig. 3 The governing unit

$$
\begin{aligned}
& U=\left(\begin{array}{c}
\rho \\
\rho u \\
\rho v \\
\rho w \\
e
\end{array}\right), \quad F=\left(\begin{array}{c}
\rho u \\
\rho u^{2}+p \\
\rho u v \\
\rho u w \\
(e+p) u
\end{array}\right), \\
& G=\left(\begin{array}{c}
\rho v \\
\rho u v \\
\rho v^{2}+p \\
\rho v w \\
(e+p) v
\end{array}\right), \quad H=\left(\begin{array}{c}
\rho w \\
\rho u w \\
\rho v w \\
\rho w^{2}+p \\
(e+p) w
\end{array}\right)
\end{aligned}
$$

The primitive variables in the unknown $U$ are density $\rho$, velocity components $u, v$ and $w$, and total energy per unit volume $e$. The equation of state for the perfect gas is given by:

$e=\frac{p}{\gamma-1}+\frac{1}{2} \rho\left(u^{2}+v^{2}+w^{2}\right)$

where $p$ is the pressure and the specific heat ratio $\gamma=1.4$.

Using the finite volume method, the equations can be converted to the integral form over the governing volume:

$\int_{\Omega} \frac{\partial U}{\partial t} \mathrm{~d} x \mathrm{~d} y \mathrm{~d} z+\int_{\Gamma}(F \mathrm{~d} y \mathrm{~d} z+G \mathrm{~d} x \mathrm{~d} z+H \mathrm{~d} x \mathrm{~d} y)=0$

where $\Omega$ and $\Gamma$ denote the cubage and boundary of the governing volume, respectively. As shown in Fig. 3, hexahedron unit is adopted as the governing volume on the unstructured mesh and governing variables are fixed at the center of the unit. The HLLC scheme is applied to compute the fluxes on the governing unit boundaries and the second-order MUSCL scheme and the first-order time integral are used to reconstruct the governing variables at the unit center [13].

The unstructured mesh is refined according to the density gradient of the flowfield and the maximum level of refinement is three. The refinement is controlled by a threshold function. If the function value exceeds the refinement threshold value, the governing unit is split into eight subunits; if the function value is less than the coarsening threshold value, the eight subunits are reunited to be one. The threshold function is given by: 
$\varepsilon=\max \left(\frac{\left|\nabla_{l} \rho\right|_{c}-\left|\nabla_{l} \rho\right|_{i}}{\alpha_{f} \rho_{c} / d l+\left|\nabla_{l} \rho\right|_{i}}, \frac{\left|\nabla_{l} \rho\right|_{c}-\left|\nabla_{l} \rho\right|_{j}}{\alpha_{f} \rho_{c} / d l+\left|\nabla_{l} \rho\right|_{j}}\right)$

where $i$ and $j$ denote two neighboring governing units, and $c$ denotes the midpoint of the boundary between these two units. $\nabla_{l}$ and $d l$ are the gradient and spatial interval from the center of unit $i$ to the center of unit $j . \alpha_{f}$ is a parameter with a small value to avoid division by zero.

In the present numerical simulations, the boundary conditions on the wedge surfaces, the upstream boundary, the downstream boundary and the mainstream boundary are set to be the slipping solid condition, the inflow condition, the outflow condition and the mirror condition, respectively. The air ahead of the incident shock wave is motionless and the air behind is calculated by the Rankine-Hugoniot relations for a given shock wave Mach number.

\section{Validation of numerical algorithms}

The numerical algorithms are validated by comparing the numerical flowfield with the experiment results. The experiment is conducted in the $100 \mathrm{~mm} \times 180 \mathrm{~mm}$ diaphragmless shock tube in the Shock Wave Research Center, Tohoku University, Japan. The shock waves are visualized with double exposure diffuse holographic interferometry. In order to show the 3D shock waves clearly, the interval between the first and second exposure is set to be $1 \mu \mathrm{s}$.

Figure 4 shows the experimental and numerical results of the 3D shock wave reflection over two perpendicularly intersecting wedges. The incident shock wave Mach number is $M_{\mathrm{s}}=2.0$ and the inclination angles of the horizontal and vertical wedges are $\alpha=43.5^{\circ}$ and $\beta=30^{\circ}$, respectively. In Fig. 4a, it is obvious that the incident shock wave (I) is reflected over the wedges and a single-Mach reflection $(R$, $\mathrm{M})$ appears over the horizontal wedge. Note that the reflected shock wave $(\mathrm{R})$ over the vertical wedge is not as clear as the one over the horizontal wedge. It is mainly because the inclination angle of vertical wedge is relatively small and the reflected shock wave over it is relatively weak. However, it is still obvious that the reflection over the vertical wedge is a MR as the Mach stem (M) is visualized clearly. In the corner of the two intersecting wedges, the two Mach stems intersect each other forming a 3D forward-leaning Mach stem $\left(\mathrm{M}^{\prime}\right)$ followed by a secondary reflected shock wave $\left(\mathrm{R}^{\prime}\right)$. Figure $4 \mathrm{~b}$ shows the numerical result, which consists of three translucent isopycnic surfaces and the isopycnic lines in all the computational boundary planes. The isopycnic surfaces denote the shock waves in the $3 \mathrm{D}$ reflection. All the wave structures, such as the incident shock wave (I), the Mach stems $(M, M)$, the reflected shock waves $(R, R)$, the secondary reflected shock wave $\left(\mathrm{R}^{\prime}\right)$ and the $3 \mathrm{D}$ Mach stem $\left(\mathrm{M}^{\prime}\right)$, can be identified clearly and agree well with the experimental result.
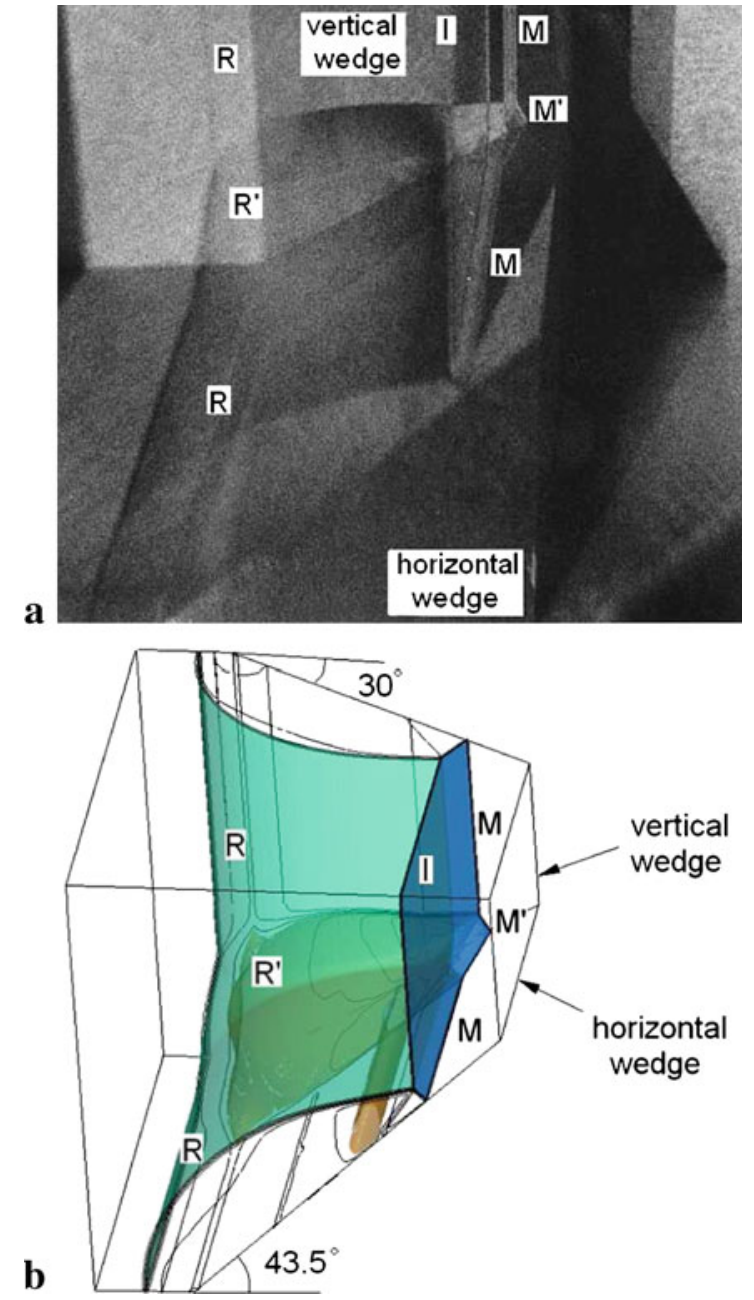

Fig. 4 Shock wave reflection over two intersecting wedges for $\alpha=$ $43.5^{\circ}, \beta=30^{\circ}$ and $M_{s}=2.0$ : a experiment result; and $\mathbf{b}$ numerical result

As the inclination angle of horizontal wedge increases to $55^{\circ}$, the pattern of the reflection over the wedge turns to a RR, as shown in Fig. 5a. It is found that the typical 3D Mach stem as depicted in Fig. 4a does not occur in the present case. Instead, the reflection of Mach stem (M) over the horizontal wedge is a RR. The secondary reflected shock wave $\left(\mathrm{R}^{\prime}\right)$ intersects the Mach stem $(\mathrm{M})$ at the reflection point, which lies on the intersecting line of the two wedges. Figure $5 \mathrm{~b}$ shows the corresponding numerical result and the same flowfield structures (I, M, R, R, R') can be visualized clearly indicating an agreement with the experiment.

In both numerical cases, the wave structures, such as the 3D Mach stems, the 2D Mach stems, the reflected shock waves and the secondary reflected shock waves, appear to be identical with the experimental results. Therefore, it is believed that the numerical method is well validated and acceptable for the present study. 

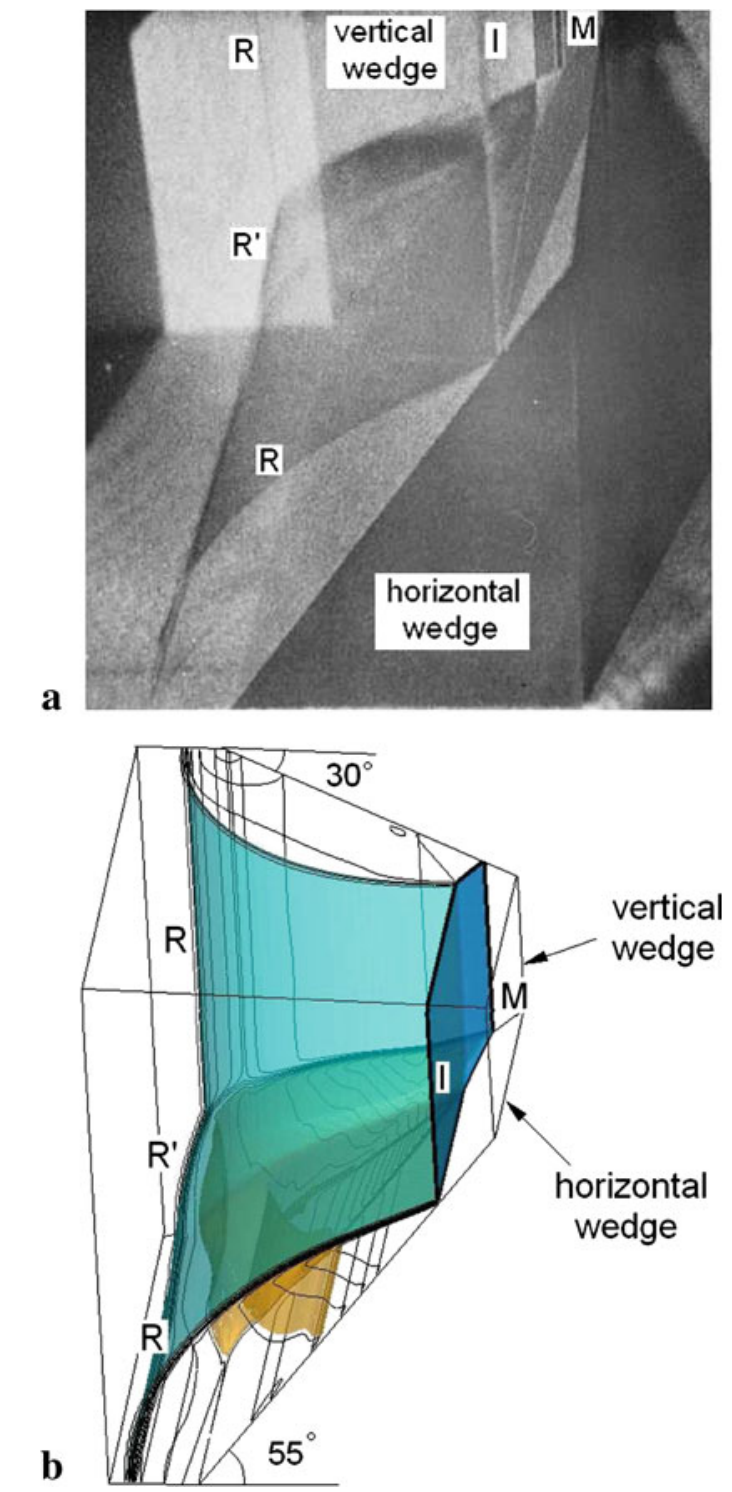

Fig. 5 Shock wave reflection over two intersecting wedges for $\alpha=55^{\circ}, \beta=30^{\circ}$ and $M_{s}=2.0$ : a experiment result; and $\mathbf{b}$ numerical result

\section{Results and discussion}

\subsection{MR-MR interaction}

The shock wave reflection for the wedge angles $\alpha=\beta=20^{\circ}$ and incident shock wave Mach number $M_{\mathrm{S}}=3.0$ is shown in Fig. 6. Both of the patterns of the reflections over the vertical and horizontal wedges are single-Mach reflections. In the corner of the two intersecting wedges, the Mach stems intersect each other and a 3D Mach stem appears. Figure 7 shows the local enlargement of the 3D Mach stem. It is obvious that the 3D Mach stem twists slightly and is approximately planar. Figure 8 shows the 3D shock reflection from a different visual angle. In this figure the shock wave configuration in the

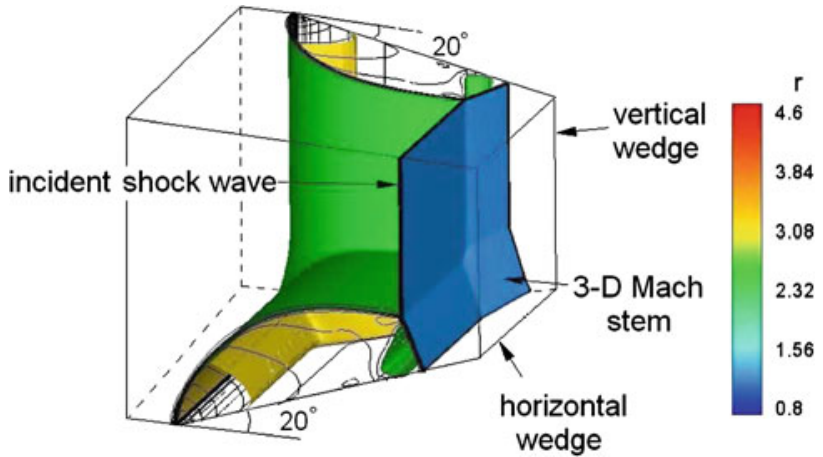

Fig. 6 Shock wave reflection over two intersecting wedges for $\alpha=\beta=20^{\circ}$ and $M_{s}=3.0$

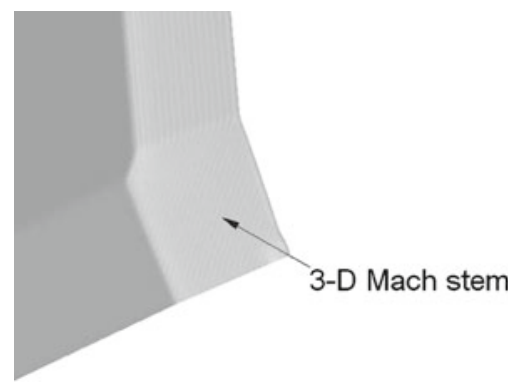

Fig. 7 The three-dimensional Mach stem for $\alpha=\beta=20^{\circ}$ and $M_{s}=3.0$

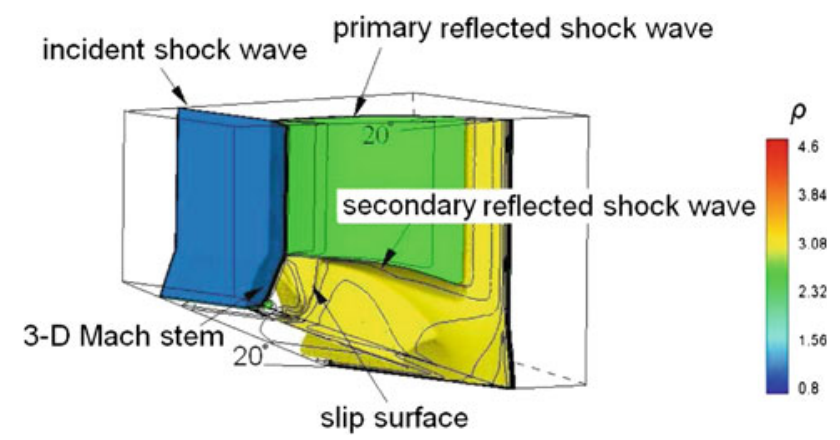

Fig. 8 Shock wave reflection over two intersecting wedges for $\alpha=\beta=20^{\circ}$ and $M_{s}=3.0$

corner of the two intersecting wedges can be observed clearly. The Mach stem on the vertical wedge is reflected over the horizontal wedge and a secondary MR appears. Similarly, the Mach stem on the horizontal wedge is also reflected over the vertical wedge and the other secondary MR appears. Accordingly, the two secondary MRs interact with each other to form the 3D Mach stem followed by the secondary reflected shock wave surface in the wedge corner. Hence, in this condition a 3D four-shock configuration forms, consisting of the incident shock wave, the 3D Mach stem, the primary reflected shock wave and the secondary reflected shock wave. Meanwhile, a 3D slip surface is observed, which is similar to the slip line structure in a 2D MR. The 3D Mach stem leans 


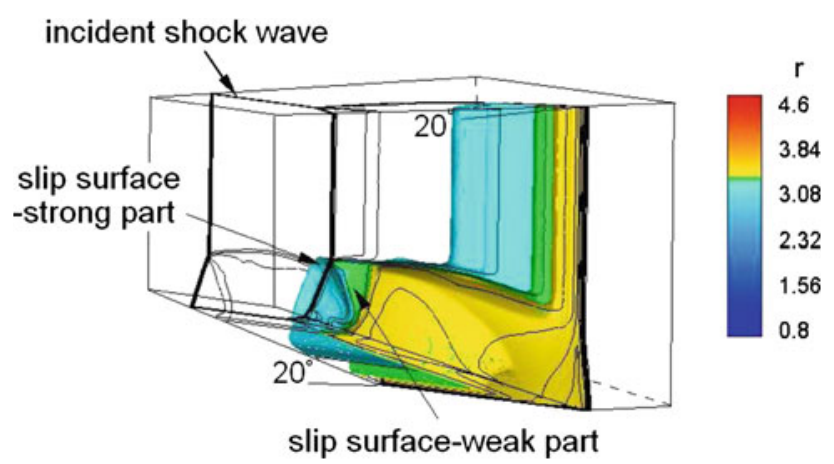

Fig. 9 The slip surface structures of shock wave reflection over two intersecting wedges for $\alpha=\beta=20^{\circ}$ and $M_{s}=3.0$

forward indicating that it has a greater shock Mach number or higher shock intensity than both of the incident shock wave and the 2D Mach stems. Thus, for the flowfield enclosed in the secondary reflected shock wave surface, density, pressure and temperature are all higher than the ones in the flowfield outside, which are identical to the ones in a $2 \mathrm{D}$ reflection case. Therefore, the complex 3D wave configuration appears inside the secondary reflected shock wave surface while the shock wave reflection still obeys the $2 \mathrm{D}$ theory in the rest flowfield.

As aforementioned, the formation of the 3D reflection configuration in the corner is the consequence of the interaction between the two secondary Mach reflections, i.e. the Mach stem on the vertical (horizontal) wedge being reflected over the horizontal (vertical) wedge. It is known that in the vicinity of the triple point of a 2D MR case, the density downstream the reflected shock wave (above the slip line) is higher than that downstream the Mach stem (below the slip line). Thus, due to the superposition of the relative high density, the density downstream the secondary reflected shock wave is much higher in the zone where the incident shock wave and the 3D Mach stem intersect than that elsewhere. As shown in Fig. 9, near the wedge surfaces the three isopycnic surfaces are apart at a distance, which means the density gradient is relatively small or the density discontinuity across the slip surface is weak. On the other hand, because of the high density in the zone where the incident shock wave intersects the 3D Mach stem, the density gradient in the vicinity is also great. Therefore, the three isopycnic surfaces are close together, which demonstrates the density discontinuity across the slip surface is relatively strong.

Figure 10 shows the shock wave reflection over two intersecting wedges with the wedge angles $\alpha=\beta=45^{\circ}$ and incident shock wave Mach number $M_{\mathrm{S}}=3$.0. Both of the patterns of the reflections over the wedges are double-Mach reflections. In the corner of the two intersecting wedges, the complex 3D reflection configuration along with 3D Mach stem appears. Figure 11 shows the local enlargement of the

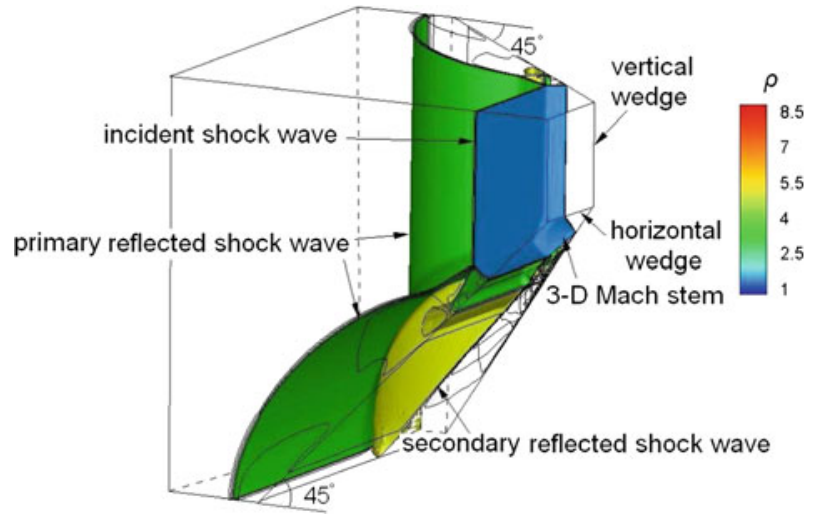

Fig. 10 Shock wave reflection over two intersecting wedges for $\alpha=\beta=45^{\circ}$ and $M_{s}=3.0$

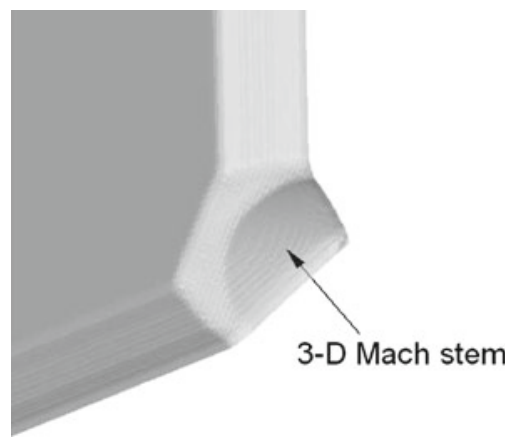

Fig. 11 The three-dimensional Mach stem for $\alpha=\beta=45^{\circ}$ and $M_{s}=3.0$

3D Mach stem. It is obvious that this 3D Mach stem twists more severely than the one shown in Fig. 7 and is no longer approximately planar. It is mainly because of the wall jet induced by the 3D compressibility in the larger inclination angle case. For a 2D case of shock wave reflection over a wedge, it has been pointed out that there is a high pressure region where the slip line approaches the wedge surface. For the reflection of a high Mach number shock wave and a large inclination angle, the high pressure drives an intensive wall jet forward and finally twists the Mach stem [14-16]. In the $3 \mathrm{D}$ case, although the shock wave Mach number is not very high, yet the two perpendicular 2D Mach reflections superimpose each other and thus the pressure is sufficiently high to generate a wall jet. Figure 12 shows the pressure profile along the intersecting line of two wedges. The abscissa is the $x$-coordinate normalized by the total length of computational region and the ordinate is the pressure normalized by the initial pressure ahead of the incident shock wave. The solid line denotes the pressure profile for $\alpha=\beta=45^{\circ}$ while the dashed line is for $\alpha=\beta=20^{\circ}$. One can see that a pressure peak appears behind the 3D Mach stem in the former case. Accordingly, an intensive wall jet is driven by the high pressure and twists the 3D Mach stem severely. As compared to the solid line, there is no obvious pressure 


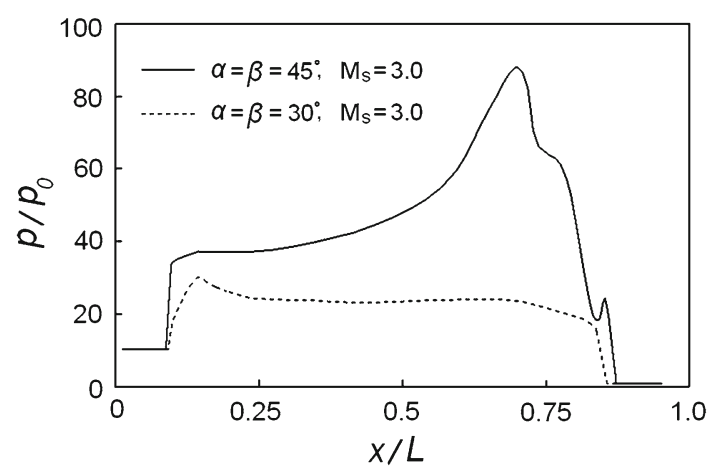

Fig. 12 Pressure profile along the intersecting line of two wedges

peak for the latter case, and thus it leads to an approximately planar Mach stem as depicted in Figs. 6 and 7.

\subsection{MR-RR interaction}

Figure 13 shows the interaction configuration over two intersecting wedges with $\alpha=52^{\circ}, \beta=45^{\circ}$ and $M_{\mathrm{s}}=3.0$. A regular reflection appears on the horizontal wedge while a double-Mach reflection appears on the vertical wedge. According to the 2D analytical method aforementioned, the Mach stem on the vertical wedge is assumed to be twodimensionally reflected over the horizontal wedge with the shock Mach number $M_{\mathrm{m}^{\prime}}=4.51$ and inclination angle $\theta_{m}=42.15^{\circ}$, as shown in Fig. 2. Coinciding with the 2D theory, such an assumed 2D reflection is a Mach reflection. The Mach stem A interacts with B resulting in a 3D Mach stem, as depicted in Fig. 14. However, the Mach stem A is much shorter as compared to the $2 \mathrm{D}$ reflection case under the same condition. This is because the horizontal wedge has a transverse inclination with regard to the Mach stem on the vertical wedge and thus it is not a complete $2 \mathrm{D}$ wedge in the secondary reflection. Actually, the velocity vector in the flowfield downstream the secondary reflected shock wave has a transverse component, which indicates this assumed $2 \mathrm{D}$ reflection has an obvious $3 \mathrm{D}$ feature.

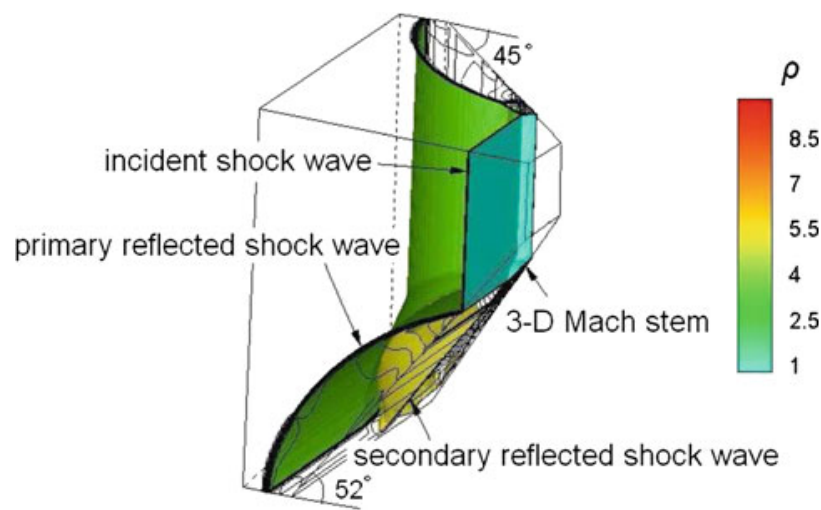

Fig. 13 Shock wave reflection over two intersecting wedges for $\alpha=52^{\circ}, \beta=45^{\circ}$ and $M_{s}=3.0$
As shown in Fig. 15 for $\alpha=55^{\circ}, \beta=45^{\circ}$ and $M_{\mathrm{s}}=3.0$, a regular reflection and a double-Mach reflection appear over the horizontal and vertical wedges, respectively. The secondary reflection of the Mach stem over the horizontal wedge, if assumed two-dimensional, should be a MR of an incident shock wave with $M_{\mathrm{m}^{\prime}}=4.51$ and $\theta_{m}=45.28^{\circ}$. However, as a matter of fact, a RR forms near the vertical wedge surface. The Mach stem A as depicted in Fig. 14 for a typical 3D Mach stem does not occur in this case. Instead, a different structure of 3D protuberance forms, as shown in Fig. 16. The new structure can be referred to as the second type of

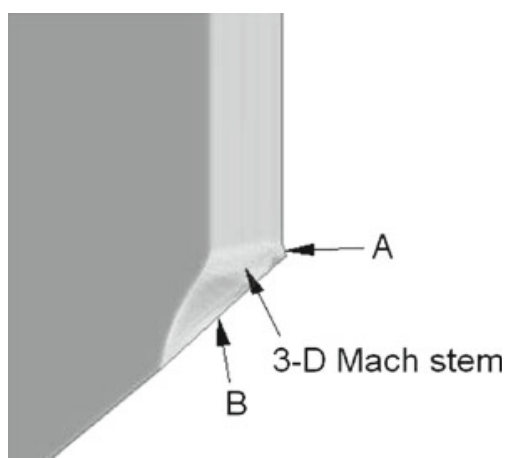

Fig. 14 The three-dimensional Mach stem for $\alpha=52^{\circ}, \beta=45^{\circ}$ and $M_{s}=3.0$

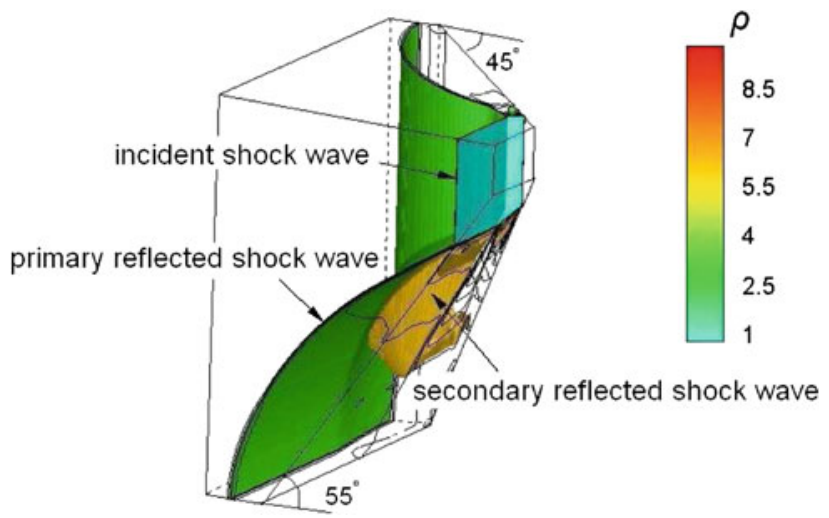

Fig. 15 Shock wave reflection over two intersecting wedges for $\alpha=55^{\circ}, \beta=45^{\circ}$ and $M_{s}=3.0$

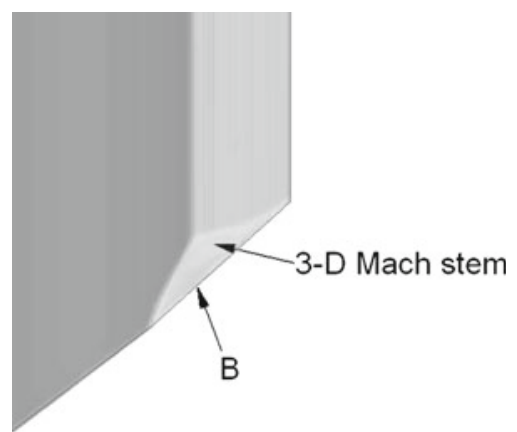

Fig. 16 The three-dimensional Mach stem for $\alpha=55^{\circ}, \beta=45^{\circ}$ and $M_{s}=3.0$ 


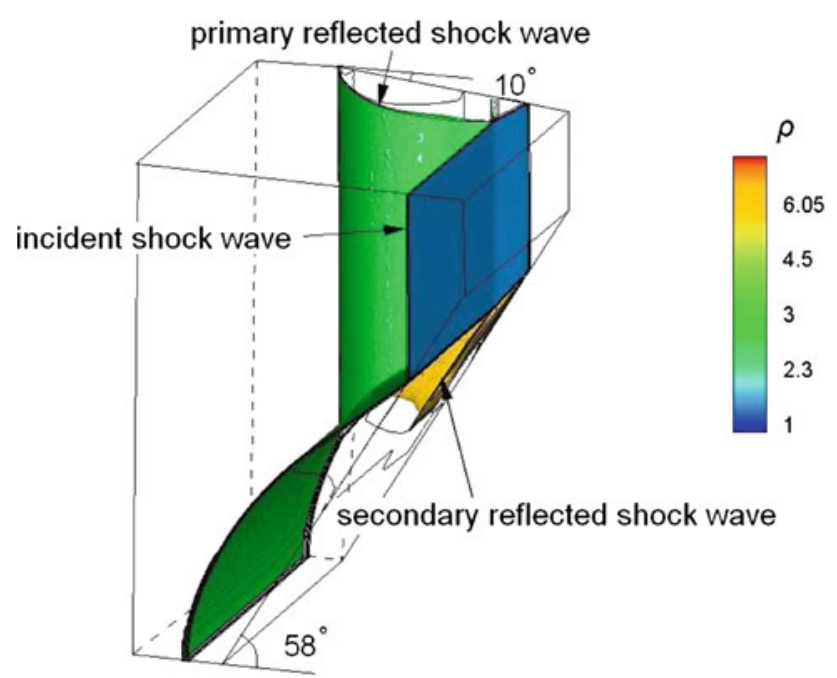

Fig. 17 Shock wave reflection over two intersecting wedges for $\alpha=58^{\circ}, \beta=10^{\circ}$ and $M_{s}=3.0$

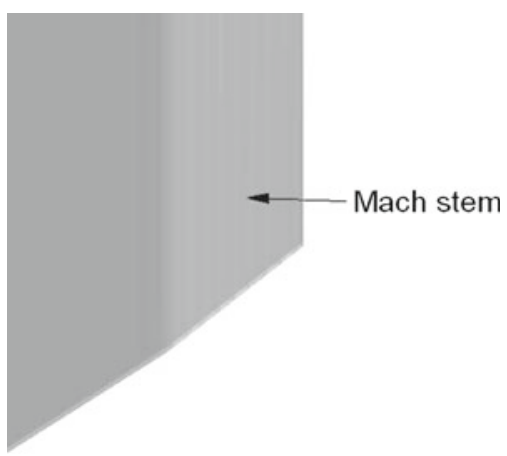

Fig. 18 There is no three-dimensional Mach stem for $\alpha=58^{\circ}$, $\beta=10^{\circ}$ and $M_{s}=3.0$

3D Mach stem while the typical one corresponds to the first type.

When $\alpha=58^{\circ}, \beta=10^{\circ}$ and $M_{\mathrm{s}}=3.0$, a regular reflection appears on the horizontal wedge and a single-Mach reflection forms on the vertical wedge, as depicted in Fig. 17. Figure 18 is the local enlargement of the flowfield in the corner. It is obvious that there is not any $3 \mathrm{D}$ protuberance structure and thus a 3D RR occurs in this case. Such a three-shock configuration consists of an incident shock wave, a primary and secondary reflected shock waves.

\subsection{RR-RR interaction}

Figure 19 shows the shock wave reflection over two intersecting wedges with the wedge angles $\alpha=\beta=55^{\circ}$ and incident shock wave Mach number $M_{\mathrm{s}}=3.0$. Two regular reflections appear respectively over the two wedges. Obviously, there is not any $3 \mathrm{D}$ protuberance structure in the corner of the two intersecting wedges. Here, the primary and secondary reflected shock waves and the incident shock wave

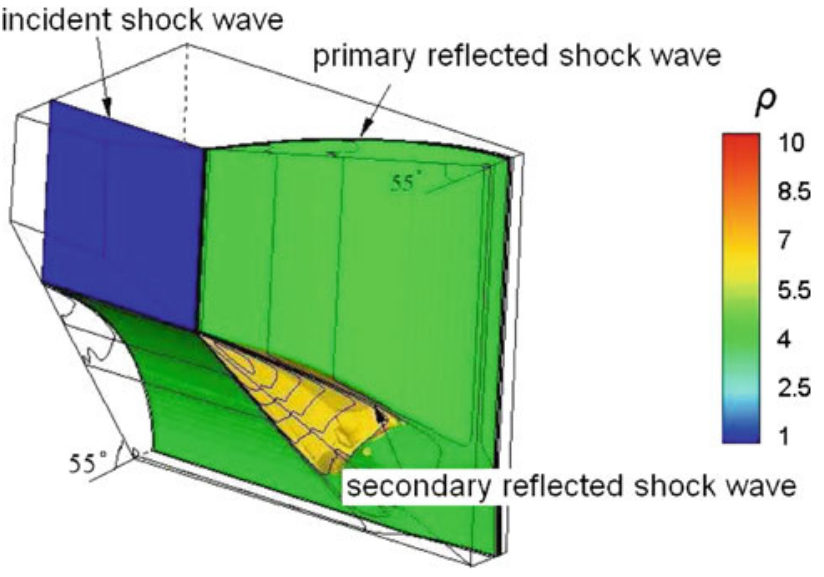

Fig. 19 Shock wave reflection over two intersecting wedges for $\alpha=\beta=55^{\circ}$ and $M_{s}=3.0$

originate from the same reflection point which always locates on the wedge intersecting line. Therefore, a three-shock configuration is formed.

\subsection{The region of 3D Mach reflection}

In summary, analogous to $2 \mathrm{D}$ shock wave reflections, 3D shock wave reflections can be generally classified into two categories, i.e., RR and MR. Using 2D shock wave reflection theory, Meguro et al [12] derived the region where the 3D Mach stem exists. However, since the secondary reflection of the Mach stem in the 3D interaction zone is not completely two-dimensional, there are limitations in their derivation. A different wave structure of 3D protuberance forms when an MR-RR interaction occurs in the corner, namely the second type of 3D Mach stem. Figure 20 shows the modified distribution of the 3D shock wave reflection pattern for $M_{\mathrm{S}}=1.5$ and $M_{\mathrm{s}}=3.0$. The ordinate and abscissa are the inclination angles of the horizontal and vertical wedges, respectively. The dashed lines, which are derived from the detachment criterion, divide the diagrams into three regions: MR-MR interaction, MR-RR interaction and RR-RR interaction. The solid lines denote the boundaries between the solution domains without 3D Mach stem and with the second type of 3D Mach stem. Meanwhile, the dashed-dotted lines separate the solutions of the first and second type of 3D Mach stem. The plots denote the numerical results in the present study. Note that with the increase of the vertical wedge angle $\beta$, the dashed-dotted lines bend upward. It is mainly because that for the same $\alpha$ the inclination wedge angle of the assumed $2 \mathrm{D}$ reflection, $\theta_{\mathrm{m}}$ [see (1)] decreases with the increase of $\beta$. Therefore, the reflection of the Mach stem over the horizontal wedge is more likely a MR so that the first type of 3D Mach stem appears. 
- with first type of 3-D Mach stem

$\Delta$ with second type of 3-D Mach stem

- without 3-D Mach stem
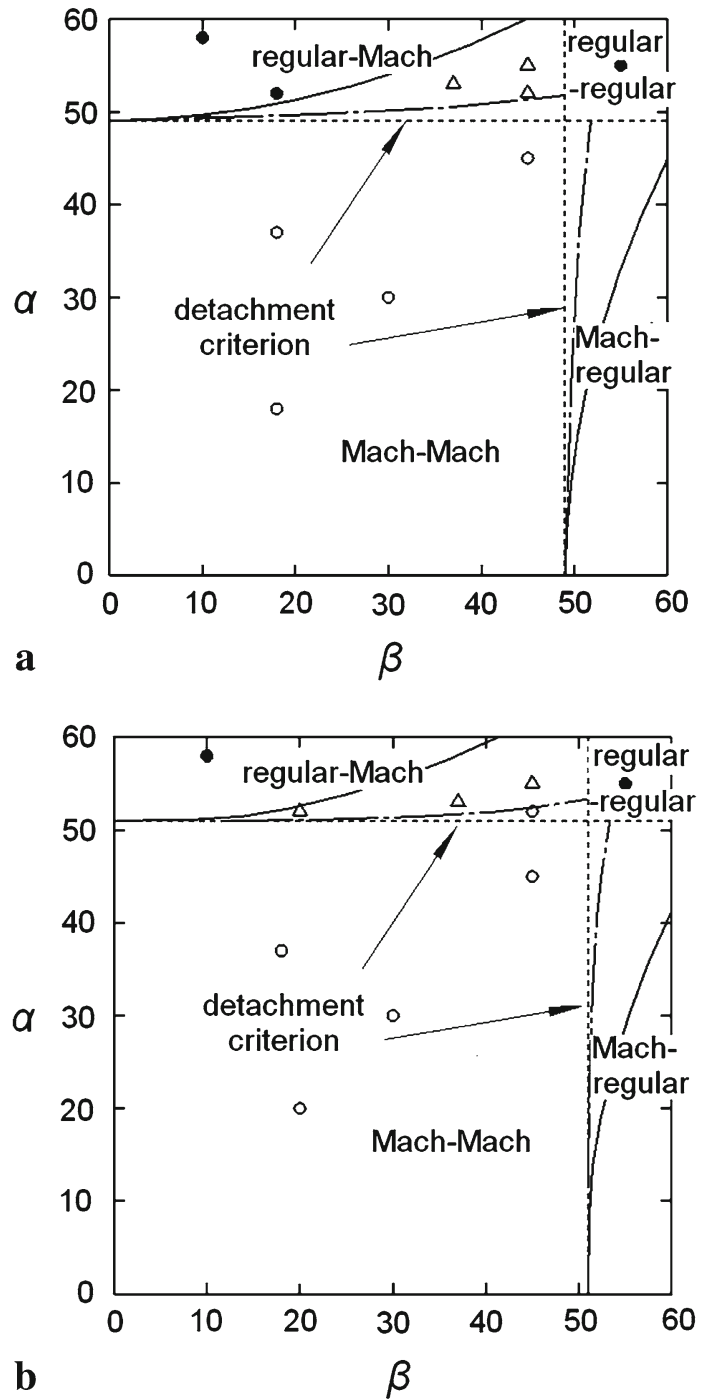

Fig. 20 The region of three-dimensional Mach stem a $M_{s}=1.5$; and b $M_{s}=3.0$

\section{Conclusions}

The numerical results can be summarized as following:

1. It is found that there are two different kinds of 3D Mach stem structures for the MR-RR interaction, namely the first and the second types of 3D Mach stem, respectively.

2. The 3D Mach stem is a twist surface. If both wedge angles are relatively small, the 3D influence is relatively weak, and the 3D Mach stem twists slightly as a result. On the other hand, if the wedge angles are relatively great, the 3D influence is obvious to twist the 3D Mach stem much severely.
3. In the corner of the two intersecting wedges, the secondary $3 \mathrm{D}$ reflected shock wave forms. Inside the surface of the secondary reflected shock wave is the $3 \mathrm{D}$ reflection zone, where complicated $3 \mathrm{D}$ wave configuration appears. Outside the secondary reflected shock wave is the 2D reflection zone where the $2 \mathrm{D}$ theory is still applicable.

4. A 3D three-shock or four-shock configuration may occur in the intersecting corner. For the MR-MR interaction, the latter forms consisting of the incident shock wave, the 3D Mach stem, the primary and secondary reflected shock waves. In the RR-RR interaction, the incident shock wave, the primary and secondary reflected shock waves meet at the same reflection point to combine a three-shock configuration. For the MR-RR interaction, either of the configurations mentioned above may appear.

5. Downstream the 3D Mach stem there is a 3D slip surface which is similar to the slip line in a 2D MR and the density discontinuity across the slip surface is not surface-wise homogeneous.

Acknowledgments The work is supported by Major Program of National Natural Science Foundation of China (90916028) and Main Direction Program of Knowledge Innovation of Chinese Academy of Sciences (KJCX2-EW-L05).

\section{References}

1. Mach, E.: Über den Verlauf von Funkenwellen in der Ebene undim Raume. Sitzungsbr Akad Wien 78, 819-838 (1878)

2. von Neumann, J.: Oblique reflection of shocks, Explos Res Rept 12. Navy Dept Bureau of ordinance, Washington, DC (1943)

3. von Neumann, J.: Refraction, interaction and reflection of shock waves, NAVORD Rep 203-45. Navy Dept Bureau of ordinance, Washington, DC (1943)

4. Hornung, H.G.: Regular and Mach reflection of shock waves. Ann. Rev. Fluid Mech. 18, 33-58 (1986)

5. Ben-Dor, G.: Shock Wave Reflection phenomena, 38-170. Springer, New York (1992)

6. Li, H., Ben-Dor, G.: Reconsideration of pseudo-steady shock wave reflections and the transition criteria between them. Shock Waves 5, 59-73 (1995)

7. Hornung, H.G., Oertel, H., Sandeman, R.J.: Transition to Mach reflexion of shock waves in steady and pseudosteady flow with and without relaxation. J. Fluid Mech. 90, 541-560 (1979)

8. Chpoun, A., Passerel, D., Li, H., Ben-Dor, G.: Reconsideration of oblique shock wave reflections in steady flows, Part 1. Experimental investigation. J. Fluid Mech. 301, 19-35 (1995)

9. Ivanov, M., Gimelshein, S., Beylich, A.: Hysteresis effect in stationary reflection of shock waves. Phys. Fluids 7, 685-687 (1995)

10. Hu, Z.M., Myong, R.S., Kim, M.S., Cho, T.H.: Downstream flow condition effects on the RR MR transition of asymmetric shock waves in steady flows. J. Fluid Mech. 620, 43-62 (2009)

11. Takayama, K.: Application of holographic interferometry to shock wave research. Proc. SPIE 398, 174-180 (1983)

12. Meguro, T., Takayama, K., Onodera, K.: Three-dimensional shock wave reflection over a corner of two intersecting wedges. Shock Wave 7, 107-121 (1997) 
13. Li, H.H.:Experimental and numerical study on unsteady complex flow and wave interaction. PhD Thesis, University of Science and Technology of China, Hefei (2005)

14. Mirels, H.: Mach reflection flow fields associated with strong waves. AIAA J. 23, 522-529 (1985)

15. Li, H., Ben-Dor, G.: Analysis of double-Mach-reflection wave configurations with convexly curved Mach stems. Shock Waves 9(5), 319-326 (1999)
16. Gao, Y.L.: Study on hypervelocity flow generation techniques and essential hypersonic phenomena. PhD Thesis, Institute of Mechanics, Chinese Academy of Science, Beijing (2008) 